\title{
An Energy Efficient Node Scheduling Algorithm of Wireless Sensor Network
}

\author{
Jie CHEN ${ }^{1,2, a}$, Xiao-Yu LI ${ }^{2, b}$, Hai-Feng ZHAO ${ }^{1,2}$,Yue-Tong CHEN ${ }^{1,2}$ \\ ${ }^{1}$ Science and Technology on Information Systems Engineering Laboratory,Nanjing, China \\ ${ }^{2}$ The $28^{\text {th }}$ Research Institute of China Electronics Technology Group Corporation,Nanjing, China \\ achenjie600006@126.com, bxyleon0708@163.com
}

Keywords: Wireless sensor network, Multi-coverage, Nodes scheduling, Energy efficient.

\begin{abstract}
After the wireless sensor network nodes deployed on the detection region. It needs scheduling algorithm to optimize nodes deployment and make full use of sensor nodes' energy consequently. This paper proposed an energy efficient nodes scheduling algorithm. It could make better use of wireless network sensors by minimize the active nodes. The algorithm can be used both in heterogeneous sensor network and homogeneous sensor network. Simulation results show that it is not only able to meet the multi-coverage requirement of the interesting region, but also can prolong the network lifetime because of balancing the sensors energy consuming.
\end{abstract}

\section{Introduction}

Wireless sensor network node energy is limited and its lifetime is short. Scheduling wireless sensor network nodes is an effective method to adjust network coverage [1], many researchers studied the sensor nodes scheduling technique and make a large deal of work recent years [2-8].However, there are many shortages: (i) these work are mainly based on binary sensor model that is when monitoring objects within the sensor's sensing radius, the probability be detected is 1 , and 0 otherwise. But sensor detections are imprecise in reality. (ii) the studies just can be used in homogeneous sensor network. However, heterogeneous sensor network's derivation is more complicated and sensor networks must consider the heterogeneity of sensor nodes. (iii) Many study results just can meet basic coverage requirement. They can't meet multi-coverage requirement.

So we provide an energy efficient node scheduling algorithm of wireless sensor network which named EK-CNSA (Efficient K-Coverage Node Schedule Algorithm). It adopted the probability sensor model and could meet k-coverage requirement of the interesting region. This algorithm can be used both in homogeneous sensor network and heterogeneous sensor network. We can configure the coverage degree of interesting region neatly by changing the value of $K$. It's an exceptional case When $K$ is 1 .

\section{EK-CNSA Detection Model}

We suppose $N$ sensor nodes deployed in the detection region with high density, this is the commonly precondition in the application of high coverage quality (battlefield e.g.). The network sensor nodes probabilistic sensor detection model is (refer with: Eq. 1):

$$
C\left(S_{i}, P\right)= \begin{cases}0, & r+r_{e} \leq d_{i P} \\ \frac{E_{i r}}{E_{i}} e^{\left(-\lambda \alpha^{\beta}+\alpha \sigma\right)}, & r-r_{e}<d_{i P}<r+r_{e} \\ 1, & r-r_{e} \geq d_{i P}\end{cases}
$$

Where re $\left(r_{e}<r\right)$ is a measure of the uncertain in sensor detection, $E_{i}$ is the initial energy of node $S_{i}, E_{i r}$ is the residual energy, $\alpha=d_{i P}-\left(r-r_{e}\right)$, and $\lambda$ and $\beta$ are parameters that measure detection probability when a target is at distance greater than $r_{e}$ but within a distance from the sensor [9].The 
$\sigma$ is a normally distributed random numbers, expresses the reality effect of disturbance on the detection probability. All sensors have equal status, no differentiation of cluster head node and ordinary node.

\section{EK-CNSA Algorithm}

EK-CNSA algorithm includes three parts: 1.K-coverage decision algorithm; 2.Nodes eligibility decision algorithm based on the result of $K$-coverage decision algorithm; 3 . Nodes scheduling algorithm based on the result of the nodes eligibility decision algorithm to schedule the nodes. The flowing text will introduce these three algorithms.

\section{$K$-coverage Decision Algorithm}

$K$-coverage decision algorithm is the key part of EK-CNSA. Computing the $K$ value is the core problem. While computing the coverage degree (to get $K$ value), We first propose the "coverage degree equal theorem", and then we convert this problem to computing coverage degrees of limited discrete points which reduces the computing cost largely. The following will introduce our method. Firstly, some definitions are brought forward.

Definition. In addition, "coverage" is defined based on Binary sensor model traditionally. We define it based on probabilistic sensor detection model newly.

Definition1. coverage. Let $\left(x_{i}, y_{i}\right)$ be the sensor $S_{i}$ position coordinate. Let $r_{s, i}$ is equivalent radius; Let $P$ is a point in interest region, and it's position coordinate is $\left(x_{P}, y_{P}\right)$. Let $d_{i p}$ is the distance between $P$ and $S_{i}$. If $d_{i P}=\sqrt{\left(x_{i}-x_{P}\right)^{2}+\left(y_{i}-y_{P}\right)^{2}}<r_{s, i}$, we say point $P$ is covered by sensor $S_{i}$. (refer with: Eq. 2):

$$
C\left(S_{i}, P\right)= \begin{cases}1, & d_{i P}<r_{s, i} \\ 0, & d_{i P} \geq r_{s, i}\end{cases}
$$

Definition2. coverage number.

(i) Coverage Number of Point $P$ : the number of sensor nodes which cover the point $P$. Let $n$ is sensors number. So, it can be denoted as following:(refer with: Eq. 3)

$$
C N(S, P)=\sum_{i=1}^{n} C\left(S_{i}, P\right)
$$

(ii) Coverage Number of whole interesting region: the minimal coverage number value of all points in the region. Let $A$ is the interesting region, it can be denoted as following: (refer with: Eq. 4)

$$
C N(S, A)=\underset{P \in A}{\operatorname{Min}}(C N(S, P))
$$

If $A$ meet $K$-coverage, that is, the value of $C N(S, A)$ is $K$.

Coverage Number Equal Theorem. Computing the $K$ value of whole interesting region is the precondition of $K$-coverage decision algorithm. The interesting region is a continuous plane area. There are infinite points in the region. So it is unfeasible to compute coverage number of all points by equation (4). Literature [9] proved that "coverage number of region is equal to the minimal coverage number value of the sensor nodes circles". We proved that "the whole region coverage number is equal to the minimal coverage number value of some points in the sensor nodes circles". Consequently, convert computing region coverage number to computing points coverage number. The following context will give the analyzing process. 


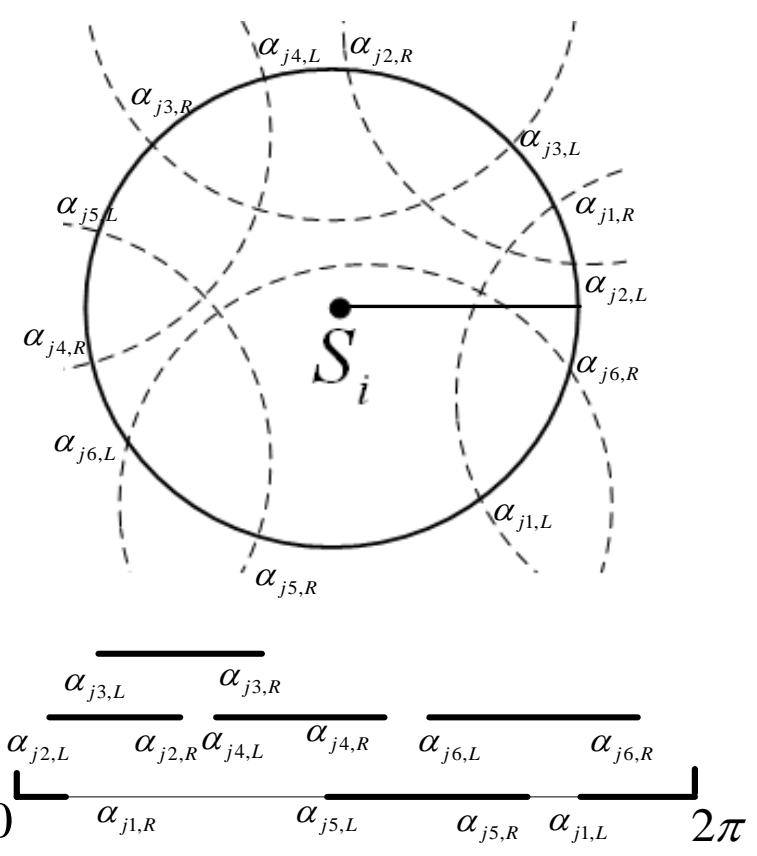

Fig. 1 node $S_{i}$ detecting circle 1-coverage

Refer with Fig. 1,the detection circle of sensor $S i$ is divided into some arcs. So, we can gain from the literature [9]:the detection circle's coverage number is equal to the minimum of the arcs' coverage number. Owing to the arc includes circular arc and two endpoints, we can know that the single arc's coverage number is equal to the minimum of circle boundary points' coverage number and "two endpoints" coverage number.

(1)Firstly, it is easy to know that the coverage number of all points in arc boundary is same (except two endpoints), so we just choose one point in arc boundary to compute the coverage number. This paper chooses the "middle point".

(2)Secondly,For the coverage number of "two endpoints", this paper provides the "coverage number equal theorem" which explains that "the minimum of the arc endpoints coverage number is equal to the minimum of arc middle point coverage number". Consequently, we just need compute coverage number of the arcs middle point to obtain the sensor circle boundary's coverage number.

Theorem. coverage number equal theorem. Supposed random two sensor nodes' locations are not superposition. The minimum of sensor circle boundary intersect points' coverage number is equal to the minimum of arc middle points coverage number.

According to the "theorem", we can use the method of computing all arcs middle point's coverage number to replace computing the sensor circles' coverage number, thus largely reduce the computing cost.

Computing the Middle Point Coordinate.Refer with Fig.2, sensors $S_{i}$ and $S_{j}$, with coordinates $\left(x_{i}, y_{i}\right)$ and $\left(x_{j}, y_{j}\right)$, Let $d_{i j}=\sqrt{\left(x_{i}-x_{j}\right)^{2}+\left(y_{i}-y_{j}\right)^{2}}, \beta_{i j}=\arctan \left(\frac{y_{j}-y_{i}}{x_{j}-x_{i}}\right)$ be the azimuth angle of $S_{i}$ and $S_{j}$.

When $S_{i}$ sensor circle and $S_{j}$ sensor circle intersecting, the radian of two intersecting points in $S_{i}$ detection circle can be separated gained from following: (refer with: Eq. 5)

$$
\begin{cases}\alpha_{j L}, \alpha_{j R}=\beta_{i j} \pm \arccos \left(\frac{d_{i j}{ }^{2}+r_{i}^{2}-r_{j}^{2}}{2 d_{i j} r_{i}}\right) & x_{j}-x_{i} \geq 0 \text { 且 } y_{j}-y_{i} \geq 0 \\ \alpha_{j L}, \alpha_{j R}=\beta_{i j} \pm \arccos \left(\frac{d_{i j}{ }^{2}+r_{i}^{2}-r_{j}^{2}}{2 d_{i j} r_{i}}\right)+\pi & x_{j}-x_{i}<0 \\ \alpha_{j L}, \alpha_{j R}=\beta_{i j} \pm \arccos \left(\frac{d_{i j}{ }^{2}+r_{i}^{2}-r_{j}^{2}}{2 d_{i j} r_{i}}\right)+2 \pi & x_{j}-x_{i} \geq 0 \text { 且 } y_{j}-y_{i}<0\end{cases}
$$




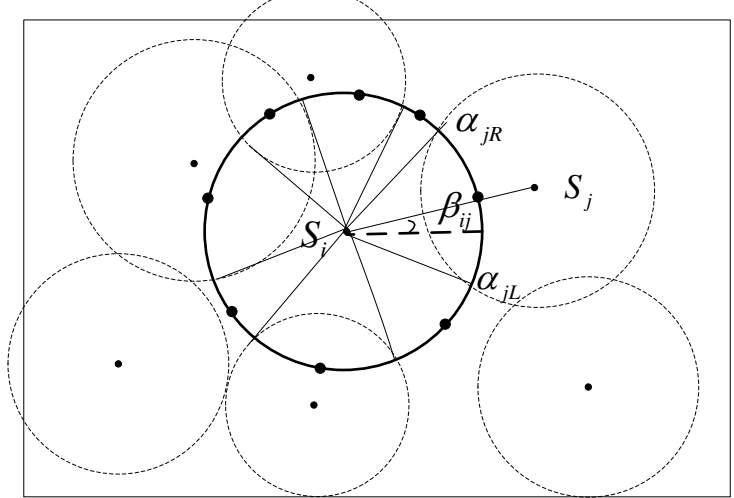

Fig. 2 node $S_{i}$ arcs and arcs middle points

Let the neighbors node set of sensor $S_{i}$ be $N\left(S_{i}\right)$,Let the number of neighbor nodes be $K(i)$.So, there are $M$ intersecting points in $S_{i}$ detection circle, $\mathrm{M}=2 \mathrm{~K}(\mathrm{i})$.Collating these intersecting points according to the radian and make a sequence. See Figure 4.The $S_{i}$ detection circle is divided to $M$ arcs by $M$ intersecting points, Let be $\left(\alpha_{i, V}, \alpha_{i, V+1}\right), \mathrm{V}=1, \ldots, \mathrm{M} . \alpha_{i, M+1}=\alpha_{i, 1+2 \pi}$ 。 Let the middle point coordinate of Arc $\left(\alpha_{i, V}, \alpha_{i, V+1}\right)$ be $P_{i, V}\left(x_{i, V}, y_{i, V}\right)$, which can be gained from following: (refer with: Eq. 6)

$$
\left\{\begin{array}{l}
x_{i, V}=x_{i}+r_{i} \cos \left(\frac{\alpha_{i, V}+\alpha_{i, V+1}}{2}\right) \\
y_{i, V}=y_{i}+r_{i} \sin \left(\frac{\alpha_{i, V}+\alpha_{i, V+1}}{2}\right)
\end{array}\right.
$$

Computing Region Coverage Number.Firstly, a single sensor node $S_{i}$ coverage number can be gained from following: (refer with: Eq. 7)

$$
C N\left(S, S_{i}\right)=\underset{P_{i, v} \in P_{i}, v=1, \ldots, M}{\operatorname{Min}}\left(C N\left(S, P_{i, v}\right)\right)
$$

Owing to the whole detection region coverage number is equal to the minimum of nodes coverage number in the wireless sensor network [9]. Let the detection region be $A$, Sensor $S_{i} \in S$, the coverage number of region $A$ can be expressed by equation (8):

$$
C N(S, A)=\underset{S_{i} \in S}{\operatorname{Min}}\left(C N\left(S, S_{i}\right)\right)
$$

We premised the sensor $S_{i}$ is intersected with other sensors in the network When using equation (8) to compute region coverage number. We classified the non-intersect into two instances: one instance is that $S_{i}$ is leaved from other sensors, that is, $d_{i j}>r_{i}+r_{j}$. In this instance, the detection region coverage number is 0 .The other instance is that the detection circle of $S_{i}$ is inside of some other sensor detection circle. In this instance, we can ignore $S_{i}$. If we calculated all the sensors in the net $S$ $=\left\{S_{1}, S_{2}, \ldots, S_{i}, \ldots, S_{n}\right\}$ meet $K$ coverage, the coverage number of whole detection region $A$ is $K$ coverage.

\section{Nodes Eligibility Decision Algorithm}

Nodes eligibility decision algorithm is the core of node sleeping schedule strategy which based on network coverage [10].The eligibility decision correctness impacts on the coverage quality and energy cost. If it determines more nodes to sleep, the coverage quality will not be achieved. If it determines less nodes to sleep, the energy will be consumed more, which can't prolong the wireless network lifetime. The node eligibility decision rule depends on different application. In this paper, our decision rule is whether the detecting region is $K$ coverage.

The eligibility means that whether the node can be active to continue to work. There are two results: eligibility or un-eligibility. If node is eligible, it is regarded as active node to continue to work. If node is un-eligible, it is regarded as sleeping node to break off.

This paper provides a distributed node eligibility decision algorithm. Refer with Eq. 7, in detecting region $A$, Every sensor node just need to collect local neighbor nodes information to make decision. The $S_{i}$ eligibility decision steps are: 
(1)Computing sensor $S_{i}$ equivalence radius according to the node detection probability and application need minimum detection probability $P_{0}$.

(2)Computing $S_{i}$ neighbor nodes net $N\left(S_{i}\right)$ according to $S_{i}$ coordinate.

(3)Computing arcs $\left\{\left(\alpha_{i}, j L, \alpha_{i}, j R\right) \mid S_{j} \in N\left(S_{i}\right)\right\}$ which intersected by $S_{i}$ and $N\left(S_{i}\right)$ and mapping the arcs into $[0,2 \pi]$.Saving the left and right endpoints of arcs into set $P_{L, R}$ (differentiating left and right endpoints ) and sequencing the $P_{L, R}$ into linked list $\mathrm{L}$.

(4)Computing the middle point coordinate using formula (6).Let all middle points set be $P_{i}$.

(5)Judging whether $S_{i}$ is boundary node, If yes, continue, otherwise go to step(7).

(6)Find out points which outside the region $\mathrm{A}$ in $P_{i}$ and make those coverage number is $\infty$.

(7)Computing the points coverage number in $P_{i}$ (according to Definition2 and Definition3) and find out the minimum, Let the minimum coverage number be $K_{\min }$. $K_{\min }$ is the $S_{i}$ detecting circle coverage number.

(8)If $K_{\min }>K$, node $S_{i}$ is un-eligible; If $K_{\min } \leq K$, node $S_{i}$ is eligible.

\section{Nodes Scheduling Algorithm}

In scheduling strategy, Nodes are differentiated into two kinds: active and sleep. The active nodes do detecting and transporting messages by wireless multi-hop. The sleep nodes don't work. Supposed sensor nodes work process into "wheel” period. At the start of "wheel” period scheduling nodes: scheduling eligibility node to work which realized coverage reality, scheduling un-eligibility node to sleep which realized energy-saving.

We provided a distributed node scheduling algorithm based on eligibility decision algorithm.

\section{EK-CNSA Algorithm}

Base on K-coverage decision algorithm, Nodes eligibility decision algorithm and Nodes scheduling algorithm, the following text will give the EK-CNSA working process.

In distributed mode, nodes work and run EK-CNSA in synchronism. There is not a concentrating processor. The sensor node just need neighbor nodes information to make decision. Refer with Fig. 3, it is the EK-CNSA process flow of a single sensor node.

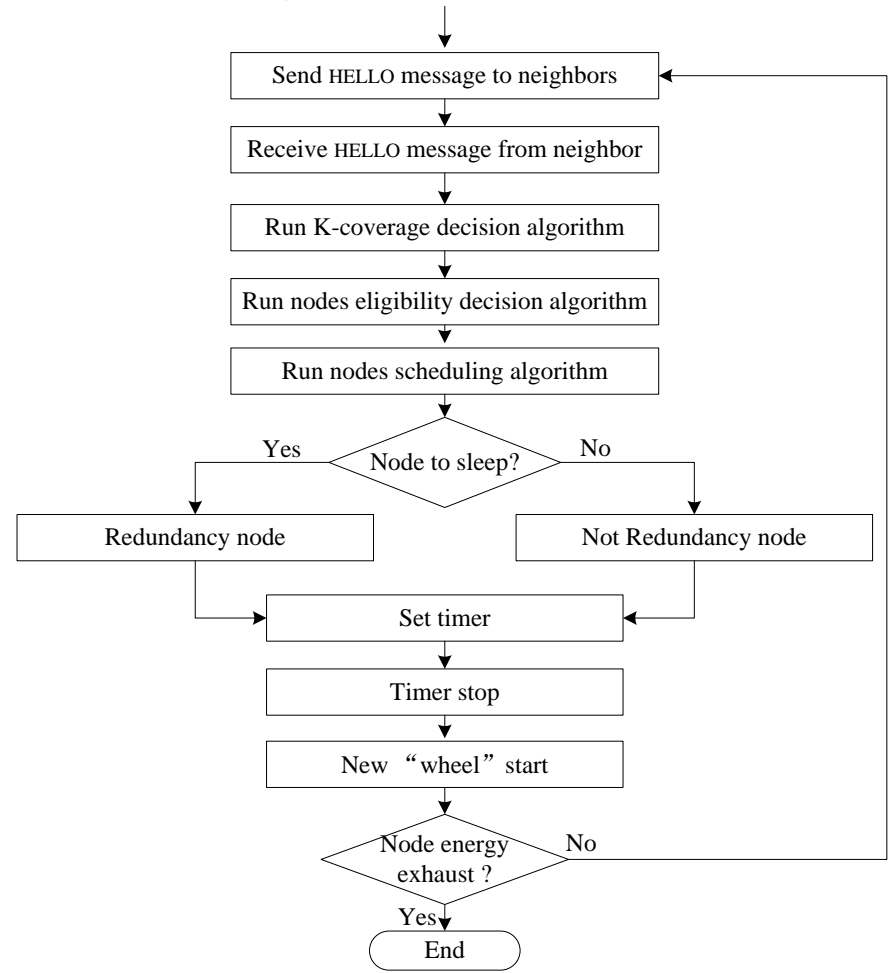

Fig. 3 sensor node running EK-CNSA flow

Because of wireless network adjusts topological structure dynamically, distributing mode has much more expansibility. 


\section{Simulation}

This paper makes simulation of EK-CNSA in energy consuming, nodes energy consuming balance and network lifetime compared with CCP (Coverage Configuration Protocol) algorithm [8]. The simulation works in an square region with $100 \mathrm{~m} \times 100 \mathrm{~m}$. The coverage number $\mathrm{K}$ is 2 .We supposed hot interesting area is $20 \mathrm{~m} \times 20 \mathrm{~m}$ which requirement coverage probability is 0.9 . The other common area requirement coverage probability is 0.7 . In the simulation running, we used three different radius (7 m, $10 \mathrm{~m}, 15 \mathrm{~m}$ ), separate node number is (75,16,3 ). At the start time, nodes with radius $7 \mathrm{~m}$ initial energy is $0.7 \mathrm{~J}$. Nodes with radius $10 \mathrm{~m}$ initial energy is $1.0 \mathrm{~J}$. Nodes with radius $15 \mathrm{~m}$ initial energy is 1.5J.

\section{Network Energy Consuming}

Supposed the energy model, initial energy and workload are the same of EK-CNSA and CCP algorithm. The figure 4 shows the energy consuming by time.

Refer with Fig. 4, we can see that EK-CNSA energy consuming is slower than CCP algorithm. The CCP network energy is used up at 580 second. However, EK-CNSA network energy is used up at 997 second.

\section{Sensor Energy Consuming Balance}

The sensor number is 94 in the simlation scene. Figure 5 shows these 94 sensors(from No.1 to No.94) energy consuming balance running EK-CNSA compared with running CCP algorithm with 300 seconds.

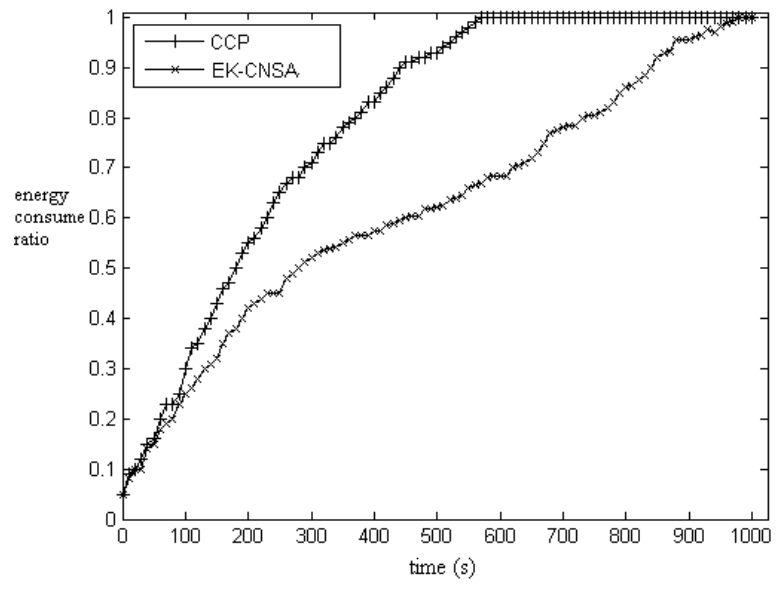

Fig. 4 Network energy consuming

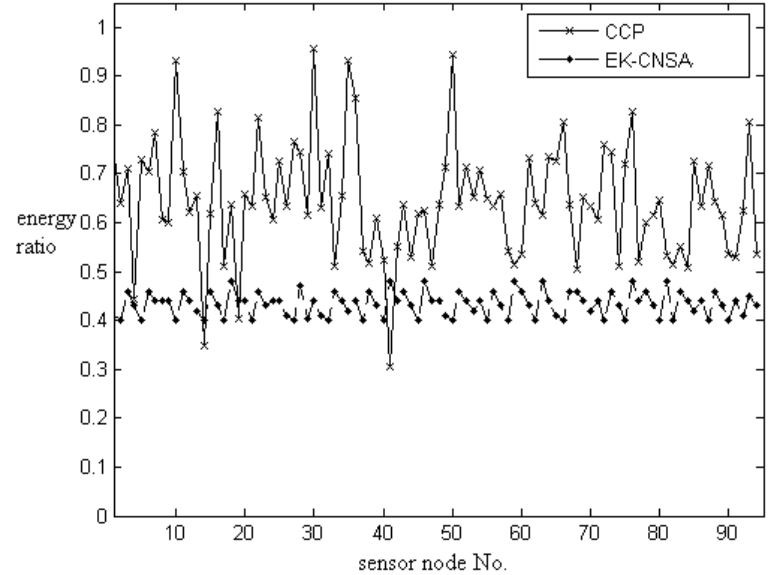

Fig. 5 Sensor energy consuming balance

Refer with Fig. 5 , we can see that sensor energy consuming between 0.3 and 0.95 using CCP algorithm. However, when using EK-CNSA algorithm, the sensor energy consuming floating in 0.4. Therefore, the network sensor energy consuming is balanced. So it can avoid sensor node died because of energy used up ahead of time.

\section{Network Lifetime}

We simulated the network lifetime using EK-CNSA and CCP. Figure 6 shows active sensors compare inner 1000 seconds. Refer with Fig.6,energy exhausted sensors appeared when using CCP algorithm at 205 second and all sensors energy exhausted at 600 second. Contrastively, energy exhausted sensors appeared when using our EK-CNSA algorithm at 500 second and all sensors energy exhausted at 995 second. It is obvious that EK-CNSA algorithm can prolong network lifetime effectively. 


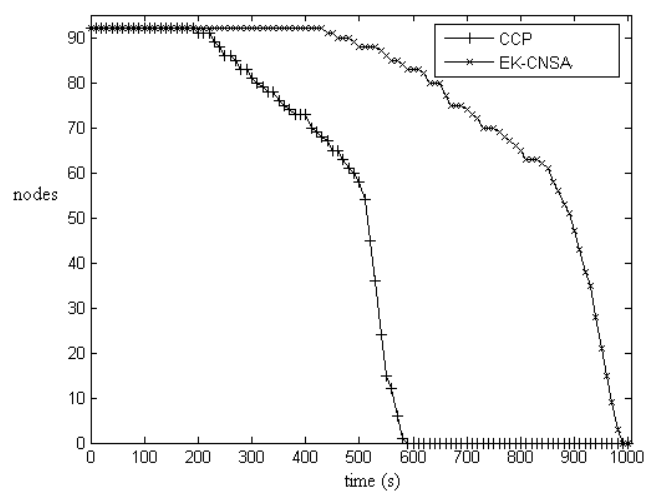

Fig. 6 Active sensor nodes by time

\section{Conclusion}

The paper first proposed K-coverage decision algorithm. Secondly, it proposed the nodes eligibility decision algorithm based on the result of k-coverage decision algorithm and proposed a nodes eligibility decision method. Thirdly, an energy efficient node scheduling algorithm based on the result of the nodes eligibility decision algorithm named EK-CNSA was proposed. EK-CNSA could make better use of sensors by minimize the active sensors. It can be used both in heterogeneous sensor network and homogeneous sensor network. Simulation results show that EK-CNSA is not only able to meet the requirement of coverage quality of the interested area, but also can prolong the lifetime of network because of balancing the sensors dissipative energy.

\section{References}

[1] FengYuan REN, HaiNing HUANG, Quang LIN. Wireless sensor network[J].Software Journal.2003,14(7) 1282 1291.

[2] Slijepcevic S, Potkonjak M. Power efficient organization of wireless sensor networks. in:ICC 2001 [C],IEEE Computer Society,2001,2:472 476.

[3] LinPing JIANG. Research on wireless sensor network coverage control.[M].ZhenJiang: Jiang Su University.2012.

[4] Cardei M,MacCallum D,Cheng X,et a1.Wireless sensor networks with energy efficient organization[J].Joumal of IntercQnnection Networks,2002,3(34):2l3 229.

[5] Wang Bang.A Survey on Coverage Problems in Wireless Sensor Networks[R].ECE Technical Report,ECE Dept,National University of Singapore,2012.

[6] Tian D,Georganas DN.A coverage-preserving node scheduling scheme for large wireless sensor networks[A].In:Proceedings of the 1st ACM international workshop on Wireless sensor networks and applications[C],ACM,2002,9:32 41.

[7] Ye F,Zhong G,Cheng J,et a1.PEAS: A Robust Energy Conserving Protocol for Long-lived Sensor Networks[A].in: Proceedings of the 23rd International Conference on Distributed Computing Systems[C],IEEE Computer Society,2003:28 37.

[8] Wang X,Xing G,Zhang Y, Lu C,et a1.Integrated Coverage and Connectivity Configuration in Wireless Sensor Networks [C]. In: ACM SIGMOBILE International Conference on Em bedded Networked Sensor Systems (SenSys2003), LosAngeles, CA, November 2003:1458 1464.

[9] Zou Y, Chakrabarty K.Sensor deployment and target localization in distributed sensor networks [J]. ACM Transactions on Embedded Computing Systems(TECS) ,2004, 3(1):61 91.

[10]Shi Bin-bin,Qin Ning-ning,Xu Bao-guo.Deployment of Energy and Distance Efficient Sensor for Wireless Sensor Networks[J].computer simulation,2009,5 (26):146-149. 